\title{
Orthogonal Constrained Meta Heuristic Adaptive Multi-View Clustering Over Multi Labeled Categorical Data Analysis
}

Srinivas Kolli ( $\sim$ srinivas6@yandex.com )

Koneru Lakshmaiah Education Foundation https://orcid.org/0000-0002-3408-5378

M Sreedevi

Koneru Lakshmaiah Education Foundation

\section{Research Article}

Keywords: Clustering, Multi labeled data, multi view clustering, optimized matrix factorization, Meta heuristic, orthogonal constraint, document clustering.

Posted Date: November 1st, 2021

DOl: https://doi.org/10.21203/rs.3.rs-917595/v1

License: (c) (i) This work is licensed under a Creative Commons Attribution 4.0 International License.

Read Full License 


\title{
Orthogonal Constrained Meta Heuristic Adaptive Multi-View Clustering over Multi Labeled Categorical Data Analysis
}

\author{
${ }^{* 1}$ Srinivas Kolli and ${ }^{2} \mathrm{M}$. Sreedevi \\ ${ }^{1,2}$ Department of CSE, Koneru Lakshmaiah Education Foundation, Vaddeswaram, Guntur, \\ Andhra Pradesh, India- 522502 \\ 1srinivas6@yandex.com
}

\begin{abstract}
In data mining, clustering is the one of the efficient research concept in real time data analysis, evaluation of attribute representation in clustering is main issue in artificial intelligence related research areas. Multi labeled clustering gives high amount of valuable data, which describes the evaluation and representation of attribute be the trending concept in multi labeled categorical data analysis. Multi dimensional clustering is combined complementary data from different dimensions to provide efficient clustering results in various conditions. Different multi view clustering techniques are proposed traditionally but they can give output as single clustering with input data. Because of multiplicity, multi dimensional data can have different grouping data which are reasonable consist perspective attributes. So how to find measurable and reasonable cluster results which are represented in multi view labeled data is still challenging task, so that in this paper, we propose a novel approach i.e. Orthogonal Constrained Meta Heuristic Adaptive Multi-View Clustering (OCMHAMVC) to represent data as a cluster with different categories. Based on multi labeled data, first proposed approach evaluates low dimensional data using optimized matrix factorization (OMF) method and clusters the similar labeled sample data into prototype cluster of dimensional data. After that we represent data in desirable orthonormality constrained view of data using adaptive heuristic to combine complementary data from different dimensions, also provide complexity in computational analysis of data representation. Experimental results of proposed approach applied on high amount of multi view data gives scalable and efficient performance with comparison to traditional multi view related clustering approaches.
\end{abstract}

Key Words: Clustering, Multi labeled data, multi view clustering, optimized matrix factorization, Meta heuristic, orthogonal constraint, document clustering.

\section{Introduction}

Because of rapid implementation of computer related technology, huge amount of data to be collected from different research areas like image processing, computer vision related fusion data, processing natural language real time data. All these data consists high dimensional features with complex structures and it explores different types of dimensions related to diverse features [1-5]. These high dimensional data describes about abundant data and curse of 
dimensionality, so managing high dimensional data in terms of concern widespread is the major issue to optimize the dimension. Reduction of dimensionality is am efficient solution for abundant data which is matched input data at low dimensional space complexity and representation of low dimensionality from hidden data with respect to input data.

Different reduction of dimensionality methods have been used for multi labeled data, based on theoretical analysis optimized matrix factorization be the research hotspot with easier implementation. Independent Component analysis (IDC) [6], Principle component analysis (PCA) [7], quantization of vector (VQ)[8] etc are the main factorization matrix related approaches which are having maximization of low - rank matrix formation from high dimensional data, from that we can efficiently extract and represent low-dimensional attribute relation from high dimensional data relations. All these approaches are not utilized with elements in matrix in the process of decomposition of matrix; it means maximization of matrix representation consist negative elements in representation of data in low-dimensions. As of late, deep learning has shown extraordinary execution in include portrayal undertakings [18-20]. Consequently, numerous analysts have brought profound learning into lattice factorization and proposed countless profound component portrayal strategies [21-27]. J. H. Ahn et al. [21] proposed multilayer nonnegative matrix factorization (MNMF). Unique in relation to customary NMF-based methodologies, MNMF decayed the coefficient grid a few times to acquire a fundamental part-based portrayal that can remove profound various leveled highlights from the first information. Also, to grow the scope of the implemented application, Trigeorgis et al. [22] semi-NMF with coordinated profound factorization to propose a profound semi-non-negative grid factorization (profound semi-NMF) strategy. Nonetheless, both MNMF and profound semiNMF just viewed as the profound decay of the coefficient network for the preparation information. For formation of new data issues, the premise grid was utilized to acquire the profound reduce the dimensional portrayal. In this manner, the premise framework straightforwardly influenced the consequences of the profound portrayal relates to lowdimensional. To explore the precise reduce-dimensional portrayal with profound of the first information framework, Zhao et al. [23] applied factorization relates to profound to the premise framework and proposed a profound NMF strategy dependent on-premise picture learning.

This problem may appear because of invariant data which is collected from different data domains/sources then that data should be represented in different views of data. This problem is solved with multi labeled clustering to represent data into different dimensions based on their representation of features and relation with multi features. Previously different types of multi view clustering related approaches are introduced but they are not discussed with optimality reduction to dimensionality in representation of multi labeled data with associative representation of features in supervised learning. Supervised learning approach is describes about labeled information based on their feature then it can easily identify the text. So it is necessary to identify unsupervised learning multi label data sources. So how to find measurable and reasonable cluster results which are represented in multi view labeled data is still challenging 
task, so a novel approach i.e. Orthogonal Constrained Meta Heuristic Adaptive Multi-View Clustering (OCMHAMVC) is proposed to represent data as a cluster with different categories. Based on multi labeled data, first proposed approach evaluates low dimensional data using optimized matrix factorization (OMF) method and clusters the similar labeled sample data into prototype cluster of data relates to multi-dimensions.

Main objectives of proposed approach as follows:

a) First we propose unsupervised multi labeled clustering which works based on orthonormality matrix factorization (which is the combination of normalization constraint and orthogonal constraint), it imposes to representation of regularities with different view of data.

b) We implement an objective model, it offers and represents minima of proposed implemented model

c) We perform experiments on several kinds of real time data sets which describes the efficiency of proposed approach with respect to traditional approaches in terms of accuracy and other parameters from multi labeled cluster data.

\section{Review of Related Work}

This section describes the relation between traditional clustering approaches which are applied on multi-labeled data.

Before, many grouping strategies on the single view data have been proposed. Ordinarily, these current single-see gathering approaches can be by and large isolated into three characterizations, specifically piece bunching approaches [22-24], supernatural grouping approaches [25-27], and subspace grouping approaches [28,29]. Part gathering approaches commonly use bit abilities to design the main commitments to a high dimensional piece space where grouping can be performed capably. For example, M. Tong et al. [25] use a Gaussian piece to design the commitments to a divided space and wire pair savvy constraints into part sorting out some way to coordinate the pattern of the gathering. Li et al. [23] use a social occasion of pre-shown bits to design the wellsprings of data and enhance the piece game plan locally to further develop bunching execution. Y. Li et al. [29] take in an optimal area bit from a social occasion of pre-decided pieces and brace the trade between part learning and grouping. For ghastly grouping draws near, they typically develop a partiality chart on the examples of information to describe the information similitude, and adventure the eigenstructure of this fondness diagram to acquire the clustering result. Existing unearthly clustering approaches [2527] spotlight the most proficient method to develop this proclivity chart.

For example, R. Zhang et al. [32] utilize the pairwise requirement data to build the inborn diagram and the punishment chart, separately. D.A. Spielman et al. [36] misuse discriminative component subspace to foster an amazing affection graph for further developing the supernatural bunching execution. D. Hidru et al. [37] fabricate a reformist bipartite diagram by mishandling 
multi-layer gets with a pyramid-style structure. Other than the above work, a help vector machine (SVM) is furthermore adequately applied in the gathering structure. C. Xu, Z. Guan et al. [40] propose a twin help vector machine (TWSVC) framework, in which they search for the bundle plane that is close to the spots of the contrasting pack and evades the characteristics of the rest gatherings. Lately, heaps of assessment practices on MVC have achieved promising execution reliant upon Non-negative Matrix Factorization (NMF) and its varieties, because the non-threat impediments think about better interpretability (Guan et al. 2020; Trigeorgis et al. 2018). The general idea is to search for an average inert factor through non-negative structure factorization among multi-see data (Liu et al. 2018; Zhang et al. 2019; 2020). Semi Non-negative Matrix Factorization (Semi-NMF), as maybe the most popular varieties of NMF, was proposed to extend NMF by relaxing up the factorized premise structure to be authentic characteristics. This preparation licenses Semi-NMF to have a more broad application truly than NMF. Besides researching Semi-NMF in MVC application interestingly, our strategy has another separation from the momentum NMF-based MVC techniques: As addressed, through the significant SemiNMF structure, we push data tests from a comparable class closer layer by layer. We get the idea from significant learning (Bengio 2019), henceforth this preparation has such a flavor. Note that the proposed method isn't exactly equivalent to the current significant auto-encoder-based MVC approaches (Andrew et al. 2019; Wang et al. 2020), in any case, we as a whole are of significant plan. One critical differentiation is that (Andrew et al. 2021; Wang et al. 2018) rely upon Canonical Correlation Analysis (CCA), which is confined to the 2-see case, while our system has no such limitation.

\section{Preliminaries}

This section describes the basic preliminaries used in proposed approach with appropriate operations.

a) Optimized Matrix Factorization (OMF) : Let s consider the multi labeled data which is presented in $A=\left\{a_{1}, a_{1}, \ldots \ldots . a_{n}\right\} \in M_{+}^{n \times d}$, here $\mathrm{n}$ is the different samples and $\mathrm{d}$ be the dimension, each dimensional feature vector $a_{j}(1 \leq q \leq n)$ which can be represented with sample n. OMF is explores to identify reduced rank based matrices within non-negative relations i.e. $H=\left\{h_{1}, h_{2}, \ldots . ., h_{d}\right\} \in Q_{+}^{d} \& W=\left\{w_{1}, w_{2}, \ldots ., w_{d}\right\} Q_{+}^{d}(k \prec \prec n \& d \prec D)_{\text {which }}$ can described as $A \square H W$. After explores matrix relations i.e. $\mathrm{H} \& \mathrm{~W}$, input data to be explored as $a_{j}=\sum_{i-1}^{d} h_{i} w_{j i}$ which is described with combination of linear matrix formation $W=\left\{w_{1}, w_{2}, \ldots . ., w_{d}\right\}_{\text {and }}$ impact factor $\mathrm{w}_{\mathrm{i}}$. So objective function of non-negative matrix formation is described as follows as Eqn. (1),

$$
\min _{H, W}\|A-H W\|_{F}^{2} \text { w.r.t } \quad H \geq 0, W \geq 0
$$


\|\|$_{F}$ be the Frobenius norm operation with different functionalities

Using Karush-Kuhn-Tucker (KKT) ruling condition with associative parameters with variables i.e. W\&H described as Eqn. (2),

$$
\begin{aligned}
& H_{i l}=H_{i l} \frac{\left(A W^{T}\right)_{i l}}{\left(H W W^{T}\right)_{i l}} \\
& W_{i j}=W_{i j} \frac{\left(H^{T} A\right)_{i j}}{\left(W^{T} H W\right)_{i j}}
\end{aligned}
$$

Factorization of deep learning described as Eqn. (3),

$$
W^{m} \simeq H_{1}^{m} W_{1}^{m} H_{1}^{m} \simeq H_{2}^{m} W_{2}^{m} \ldots \ldots \ldots H_{1-2}^{m} \simeq H_{l-1}^{m} W_{l-1}^{m}, H_{l-1}^{m} \simeq H_{l}^{m} W_{l}^{m}
$$

Where $H_{1}^{m}, H_{2}^{m}, \ldots ., H_{1-1}^{m}, H_{1}^{m} \& W_{1}^{m}, W_{2}^{m}, \ldots ., W_{1-1}^{m}, W_{1}^{m}$ described as co-efficient and basis matrices for $\mathrm{m}$-th dimensionality. Combining above equations are described as Eqn. (4),

$$
\min _{W_{l}^{m}, H_{l}^{m}}\left\|A^{m}-H_{l}^{m} W_{l}^{m} W_{l-1}^{m}, \ldots ., W_{2}^{m} W_{l}^{m}\right\|_{F}^{2} \text { w.r.t } \quad H_{l}^{m} \geq 0, W_{l}^{m} \geq 0
$$

Based on multiple perspectives with objective functionalities is described as Eqn. (5),

$$
\min _{W_{l}^{m}, H_{l}^{m}} \sum_{m-1}^{M}\left\|A^{m}-H_{l}^{m} W_{l}^{m} W_{l-1}^{m}, \ldots ., W_{2}^{m} W_{l}^{m}\right\|_{F}^{2} \text { w.r.t } \quad H_{l}^{m} \geq 0, W_{l}^{m} \geq 0
$$

Objective functionality with multiple attribute is describes as Eqn. (6),

$$
\min _{H_{l}^{m}, W_{l}^{m}}\left\|A^{m}-H_{l}^{m} W_{l}^{m} W_{l-1}^{m}, \ldots ., W_{2}^{m} W_{l}^{m}\right\|_{F}^{2} \text { w.r.t } \quad H_{l}^{m} \geq 0, W_{l}^{m} \geq 0
$$

\section{b) In-depth factorization of deep matrix indices}

To define or explore structure relates to complex operations and eliminate irrelevant data present in matrix A with associative modularity's. Deep learning of optimized matrix functionalities is described as Eqn. (7),

$$
\begin{gathered}
A \simeq Z_{1} W_{1} A \simeq Z_{1} Z_{2} W_{2} \ldots \ldots \ldots \ldots \ldots \\
A \simeq Z_{1} Z_{2} \ldots Z_{m} W_{m}
\end{gathered}
$$


$Z_{l} \in Q^{K_{t-1} \times K_{t}}$ be the $l-t h(l \leq m)$ matrix relates to basis, \& $W_{l} \in \mathfrak{R}^{k_{i} \times n}(>0)$ is the representation of 1th layered matrix formation, by taking this relation i.e. $Z_{l}, \ldots . .,\left(Z_{1}, Z_{2}, Z_{t}\right) \in \mathfrak{R}^{d \times k_{i}}$ be the grouped cluster formation or individually identified factorization of matrix formation with $\mathrm{m}$-dimensional layers. So that representation of different data sets with same group operations with different perspectives on the view of multi labeled data. So deep matrix factorization method is applicable to handle multi labled clustering with multiple attribute relations. Based on these preliminaries, we propose a novel heuristic model to explore multi labeled data clustering with augmented matrix formation.

\section{Proposed Model}

This section discuss about proposed approach with unsupervised learning clustering approach i.e. OCMHAMVC, this approach associate with constraints relates to orthogonal \& combined framework with co-regularization. We first describe the multi objective functions of proposed and then explore optimized maximization approach to cluster multi labeled data. Finally we analyze the computational analysis of proposed method with efficient complexity.

\section{a) Multi objective functions of OCMHAMVC}

Let us consider efficient multi labeled data set $\left\{A^{u} \in \mathfrak{R}^{m_{u} n}\right\}_{u=1}^{n_{u}}$, it consists group of sample $\mathrm{n}$ classes with different $n_{u}$ multi labels, $\mathrm{n}$ be the samples and $\mathrm{m}_{\mathrm{u}}$ be the dimensionality in $\mathrm{u}^{\text {th }}$ dimensional data. For example based on attribute data, labeled data consist 1 samples and unlabeled data consists 1-1 samples, labeled constraints are represented in multi views as Eqn. (8),

$$
X=\left(\begin{array}{cc}
C_{c^{*} l} & 0 \\
0 & I_{n-1}
\end{array}\right)
$$

$C_{i j}$ be the attribute data with ith and jth classes $c_{i j}=0$ then it labeled data with 1 samples, unlabeled sample $1_{n}-1$ defines $(n-1)(n-l)$ related to formation of identity matrix. If samples of unlabeled data are higher than threshold cluster data then using identity matrix explores the labeled constraint data from unknown labeled data. For example sample data $(1+1)$ is not defined to implemented cluster, labeled matrix data formation is described as Eqn. (9),

$$
X=\left(\begin{array}{ccc}
C_{c^{*} l} & 0 & 0 \\
0 & 1 & 0 \\
0 & 0 & I_{n-l-1}
\end{array}\right)=\left(\begin{array}{cc}
C_{c^{*} l} & 0 \\
0 & I_{n-1}
\end{array}\right)
$$


We describe auxiliary matrix $\mathrm{Z}$, for dimension represents with sample data, multiple objective functions for orthogonal matrix formation described as below,

$$
M O_{F}=\sum_{u=1}^{n_{u}} \theta_{u}\left\|A^{u}-V^{u}\left(Z^{u}\right)^{T} X^{T}\right\|_{F}^{2}+\lambda \sum_{u=1}^{n_{u}}\left\|F O\left(Z^{u}\left(Z^{u}\right)^{T}\right)-I\right\|_{F}^{2}+\sum_{u=1}^{n_{u}} \sum_{s=1}^{n_{u}} \frac{1}{2} \theta_{u s}\left\|Z^{u}-Z^{s}\right\|_{F}^{2}
$$

from this multi-objective function, explore low-dimensional feature representations for executed sample data with dimension. For each and every dimension explore representation of desirable attribute. Desirable feature representation consists two basic properties i.e. attributes between classes consists efficient discriminating factor and scalability of all the selected features should be similar. To satisfy representation of desirable feature presentation, use orthogonal constraints in multi labeled clustering. Orthogonal constraint consists low-dimensional feature representation with associative clustering prototype with different parametric notations, for efficient orthogonal constraint on discriminating factor between classes and attributes. So migrating the performance of orthogonal constraints with joint constraint matrix is described as Eqn. (10),

$$
F_{i j}\left\{\begin{array}{ccc}
1 & j=i & 1 \leq j, i \leq c \\
0 & \text { otherwise } & 0
\end{array}\right.
$$

Then orthogonal constraint framework described as $\lambda \sum_{u=1}^{n_{u}}\left\|F O\left(Z^{u}\left(Z^{u}\right)^{T}\right)-I\right\|_{F}^{2}$

Here o represents multiplication operator with different notations and then identify the controllability of orthogonal constraint relation. This is the representation of different forms in different dimensions, which can contain unity data under the structure of clustering specification.

\section{b) Convex Feature Optimization}

Based on the objective function which explores different variables $V^{u} \& Z^{u}$ applied together in objective non-convex functions based on global minima relations. Based on updated constraints update the optimization calculation, i.e. one attribute relation is associated with other attributes which are present as constant. For non-negative attribute relations with convex optimization based on Lagrange multipliers present in Lagrange matrices incorporate non-negative matrix relations are described as Eqn. (11),

$$
\begin{aligned}
& L a_{-} r=\sum_{u=1}^{n_{u}} \theta_{u}\left\|A^{u}-V^{u}\left(Z^{u}\right)^{T} X^{T}\right\|_{F}^{2}+\lambda \sum_{u=1}^{n_{u}}\left\|F O\left(Z^{u}\left(Z^{u}\right)^{T}\right)-I\right\|_{F}^{2}+\sum_{u=1}^{n_{u}} \sum_{s=1}^{n_{u}} \frac{1}{2} \theta_{u s}\left\|Z^{u}-Z^{s}\right\|_{F}^{2} \\
& +\sum_{u=1}^{n_{u}} \operatorname{tr}\left(\beta^{u}\left(V^{u}\right)^{T}\right)+\sum_{u=1}^{n_{u}} \operatorname{tr}\left(\alpha^{u}\left(Z^{u}\right)^{T}\right)
\end{aligned}
$$

By applying KKT conditions on implemented multi labeled attributes described as Eqn. (12), 


$$
\begin{aligned}
& \left(A^{u} X Z^{u}-V^{u}\left(Z^{u}\right)^{T} X^{T} X Z^{u}\right)_{i j} v_{i j}^{u}=0 \\
& \theta_{u} X^{T}\left(A^{u}\right)^{T} V^{u}-\theta_{u} X^{T} X Z^{u}\left(V^{u}\right)^{T} V^{u}-2 \lambda\left(F o\left(Z^{u}\left(Z^{u}\right)^{T}\right) Z^{u}+2 \lambda Z^{u}-4 \lambda F\left(Z^{u} \cdot Z^{u} \cdot Z^{u}\right)\right. \\
& +4 \lambda F\left(Z^{u} \cdot Z^{u} \cdot Z^{u}\right)-\sum_{s=1}^{n_{u}} \theta_{u s} Z_{j i}^{s} Z_{j i}^{u}=0
\end{aligned}
$$

Final obtaining multi-objective function is described as Eqn. (13),

$$
\begin{aligned}
& v_{j i}^{u} \leftarrow v_{j i}^{u} \frac{\left(A^{u} X Z^{u}\right)_{j i}}{\left(V^{u}\left(Z^{u}\right)^{T} X^{T} X Z^{u}\right)_{j i}} \\
& z_{j i}^{u} \leftarrow z_{j i i}^{u} \frac{\left(\theta_{u} X^{T}\left(A^{u}\right)^{T} V^{u}+2 \lambda Z^{u}+4 \lambda F\left(Z^{u} \cdot Z^{u} \cdot Z^{u}\right)+\sum_{s=1}^{n_{u}} \theta_{u s} Z^{s}\right)_{j i}}{\left(\theta_{u} X^{T} X Z\left(V^{u}\right)^{u} V^{u}+2 \lambda\left(F o\left(Z^{u}\left(Z^{u}\right)^{T}\right)\right) Z^{u}+4 \lambda F\left(Z^{u} \cdot Z^{u} \cdot Z^{u}\right)+\sum_{s=1}^{n_{s}} \theta_{u s} A^{u}\right)_{j i}}
\end{aligned}
$$

Based on above formation of objective algorithm to be described as follows:

I/p: Multi labeled data set $\left\{A^{1}, A^{2}, \ldots \ldots ., A^{n_{u}}\right\}$, no. of clusters, no. of samples, different parameters $\theta_{v}, \theta_{v s}$

1. Built constraint labeled matrix $\mathrm{X}$

2. Built optimized constraint matrix $F$

3. for $\mathrm{u}=1-\mathrm{n}$ then

a. factors with normalization i.e. $A^{u}\left(\left\|A^{u}(:, j)\right\|^{2}\right)$;

b. update initialize parameters $\mathrm{V}^{\mathrm{u}} \& \mathrm{Z}^{\mathrm{u}}$ in ploted range $[1,0]$

4. E-for

5. For for $u=1 \circledast n_{u}$ then

a. Perform matrix formations based on iterations $<\mathrm{T}$

b. Constant $\mathrm{V}^{\mathrm{u}}$ then update $\mathrm{Z}^{\mathrm{u}}$

c. Constant $\mathrm{Z}^{\mathrm{u}}$ then update $\mathrm{V}^{\mathrm{u}}$

6. E-for

7. Evaluate representation of low dimensional data i.e. $\mathrm{U}^{\mathrm{v}}=\mathrm{XZ} \mathrm{u}^{\mathrm{u}}$ 
8. Evaluate final representation of low dimensional data $U^{*}=\frac{\sum_{u=1}^{n_{u}} U^{u}}{n_{v}}$

o/p: Final multi labeled cluster result

Algorithm 1. Proposed optimization multi labeled clustering approach.

Using this algorithm, convergence of multi dimensional data clustering.

\section{c) Multi labeled Dimensional Clustering}

Based on the similarity measure, we formulate the multi label clustering with basic cluster functions, first cluster function; we evaluate average similarity weight measure from input documents which are from same cluster. Architecture to explore multi dimensional document clustering described in figure 1 .

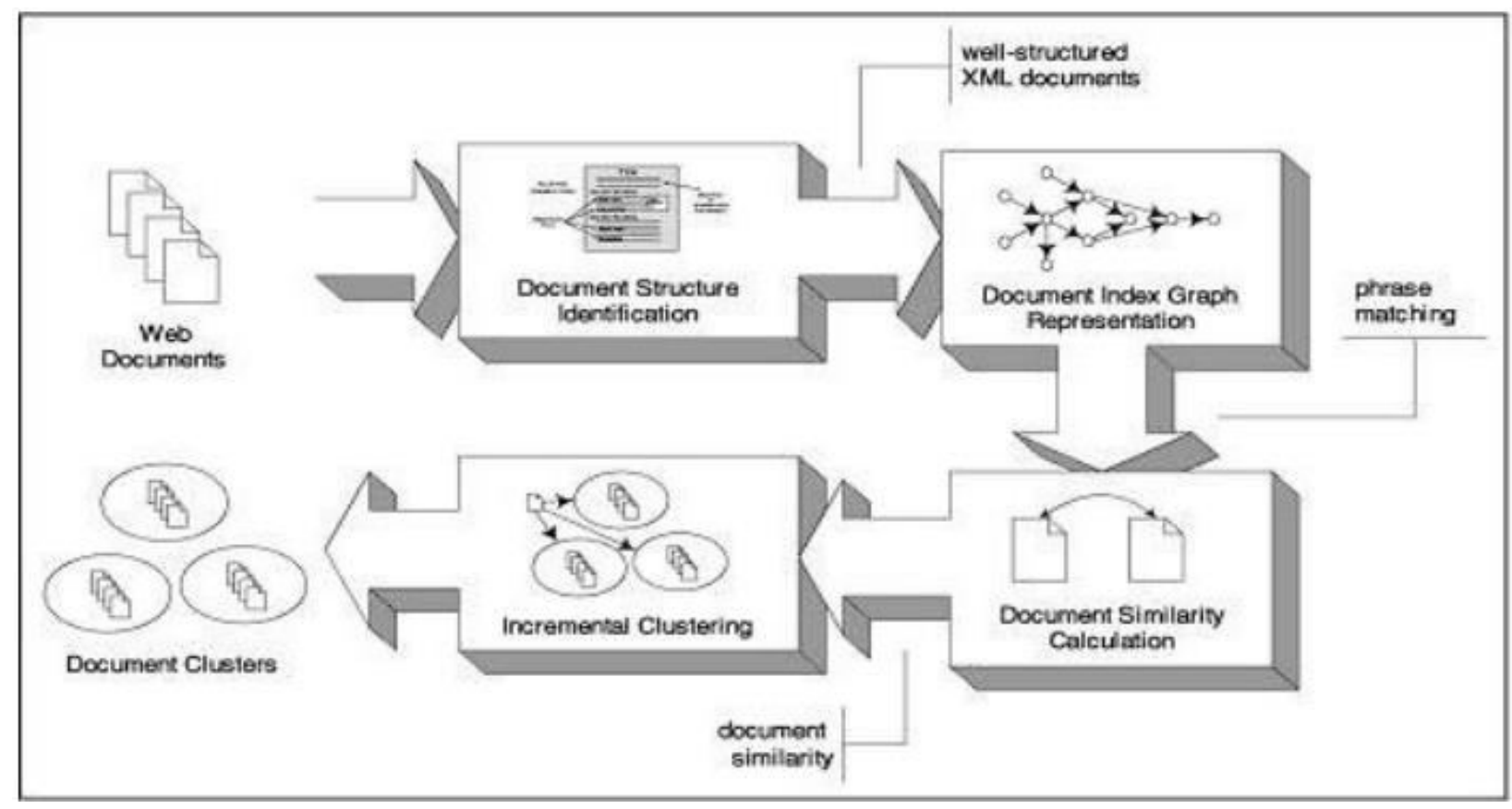

Figure 1 Architecture of proposed approach

Figure 1 describes the overall step by step procedure to collect multi dimensional cluster results. Based on this procedure, weighted similarity calculated expression as Eqn. (14), 


$$
C S=\sum_{r=1}^{n} m_{r}\left[\frac{1}{m_{t}^{2}} \sum_{t_{i}, t_{j} S_{r}} S\left(t_{i}, t_{j}\right)\right]
$$

$\mathrm{m}$ be the no.of documents, $\mathrm{n}$ be class labels, we would like to optimize the functionality to find out the similarity function as Eqn. (15),

$$
\begin{aligned}
& \sum_{t_{i}, t_{j} \in S_{r}} S\left(t_{i}, t_{j}\right)=\sum_{t_{i}, t_{j} \in S_{r}} t_{i}^{d}, t_{j}-\frac{2 m_{r}}{m-m_{r}} \sum_{t_{i} \in S_{r}} t_{i}^{t} \sum_{t_{m} \in S \backslash S_{r}} t_{h}+n_{r}^{2} \\
& =T_{r}^{d} T_{r}-\frac{2 m_{r}}{m-m_{r}} T_{r}^{d}\left(T-T_{r}\right)+m_{r}^{2} \\
& \frac{m+m_{r}}{m-m_{r}}\left\|T_{r}\right\|^{2}-\frac{2 m_{r}}{m-m_{r}} T_{r}^{d} T+m_{r}^{2}
\end{aligned}
$$

Filtering the similarity maximizing the document relations with weighted cluster functions as Eqn. (16),

$$
C F=\sum_{r=1} \frac{1}{m_{r}}\left[\frac{m+m_{r}}{m-m_{r}}\left\|T_{r}\right\|^{2}-\left(\frac{m+m_{r}}{m-m_{r}}-1\right) T_{r}^{d} T\right]
$$

Compare to maximized weighted cluster functions, $T_{r}^{d} T$ be the min-max functionality between two terms of input documents, it evaluate the based on maximized weighted attribute. Integrate the functionality of cluster related documents are expressed as Eqn. (17),

$$
\overline{C F}=\sum_{r=1}^{k} \frac{\lambda_{r}}{m_{r}}\left[\frac{m+m_{r}}{m-m_{r}}\left\|T_{r}\right\|^{2}-\left(\frac{m+m_{r}}{m-m_{r}}-1\right) T_{r}^{d} T\right]
$$

Optimized functionality (Y) for weighted functionality for related document clustering is evaluated as Eqn. (18),

$$
Y=\sum_{r=1}^{k} \sum_{t_{i} \in S_{r}} \frac{1}{m-m_{r}} \sum_{t_{h} \in S \backslash S_{r}} S\left(t_{i}-t_{h}, \frac{C_{r}}{\left\|C_{r}\right\|}-t_{h}\right)
$$

Based on similarity behind cluster formation with multiple multi label functionalities, we describe the optimization of multi labeled clustering with weighted cluster functions mainly depends on cluster functionality to be evaluated as Eqn. (19), 


$$
M L C_{o p t}=\sum_{r=1}^{k} I_{r}\left(m_{r} T_{r}\right)
$$

During this optimized multi labeled clustering with different sources, create optimized cluster results and then update convergence cluster matrix relations with similarity measure based on number of iterations applied on different input label data.

\section{Experimental Evaluation of OCMHAMVC}

This section describes the performance of OCMHAMVC with comparison to traditional approaches in terms of different parameters. Verify the proposed approach advantages with experimental document data, OCMHAMVC used similarity measure with different weighted cluster functions for multi labeled document clustering, similarity measure clustering functions works based on Euclidean distance similarity of cosine similarity and relative jacquard coefficient measurement.

\section{a) Input clustering data}

The data used for multi labeled document clustering consists real time benchmark data sets, earlier we use k1b, Reuter's 8-10 versions for document clustering, from this data, in this experiment, and we include other benchmark data sets from efficient and exhaustive data sources. Measurable similarities of downloaded data sets which are together with cloud related data sources, by using these data sets perform real time clustering applications. Mainly in our proposed approach, we use BBC Seriesdataset, Reuter's dataset, Series 3 sources dataset and MSRC dataset (http://mlg.ucd.ie/datasets/segment.html., http://mlg.ucd.ie/datasets/3sources.html, $\quad$ http://lig-membres.imag.fr/grimal/data.html, http://www.vision.caltech.edu/Image Datasets/Caltech101/., https://pgram.com/dataset/msrc-v1/. ) which are having lots of data in terms of HTML documents with different input data sources (entertainment, politics, and sports, medical and business related applications). Proposed approach performed on all these datasets and evaluates the performance evaluation with different traditional multi dimensional clustering approaches MultiNMF (local graph regularization with nonnegative matrix factorization based Multi-view clustering via joint) [1], GMNMF (multiview non-negative matrix factorization) [2] , CoNMF-P (Comment-based non-negative matrix factorization) [3] , MVCC (Multi-view clustering via concept factorization)[4] •

\section{b) Setting of Experiments}

With comparison of traditional approaches with proposed approach in terms of different parameters from their original papers, different samples are taken from data sources randomly with removal labeled data from input data, from all input data, search parameter weight with different notations. In our experiments, we use multi labeled dimensional clustering based on similarity weight measure with Euclidean distance metrics. By applying these metrics in our proposed approach with evaluate the performance of each cluster data set in evaluation of different metrics like accuracy, normalization factor, jacquard coefficient,precision, recall, fscore and performance of computational cost and memory utilization. Figure 2 describes the 
performance of proposed approach with traditional approaches in terms of multi label precision in document retrieval.

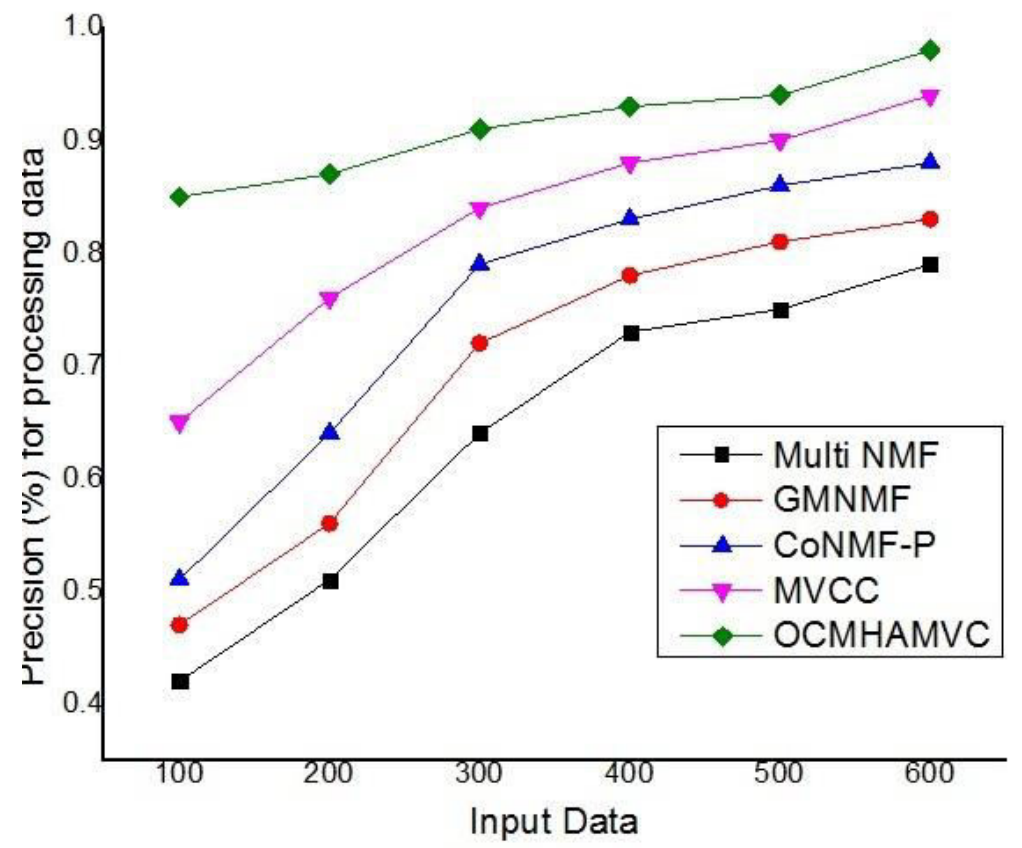

Figure 2 Performance of precision with respect to multi class label parameters.

Figure 2 show the precision performance of five different clustering approaches applied on 100$500 \mathrm{html}$ text documents with high preference of multi labeled data collection. Clustering results are presented in different way based on precision values shown in table 1, for each data set present in row, best value is represented in bold and remaining values are second best results of different approaches.

Table 1 Multi dimensional cluster results in precision

\begin{tabular}{|c|c|c|c|c|c|}
\hline $\begin{array}{c}\text { Input } \\
\text { Data }\end{array}$ & $\begin{array}{c}\text { MultiN } \\
\text { MF }\end{array}$ & GMNMF & CoNMF-P & MVCC & OCMHAMVC \\
\hline 100 & 0.42 & 0.47 & 0.51 & 0.65 & 0.85 \\
\hline 200 & 0.51 & 0.56 & 0.64 & 0.76 & 0.87 \\
\hline 300 & 0.64 & 0.72 & 0.79 & 0.84 & 0.91 \\
\hline 400 & 0.73 & 0.78 & 0.83 & 0.88 & 0.93 \\
\hline 500 & 0.75 & 0.81 & 0.86 & 0.9 & 0.94 \\
\hline 600 & 0.79 & 0.83 & 0.88 & 0.94 & 0.98 \\
\hline
\end{tabular}

Table 2 Multi dimensional clustering values in recall

\begin{tabular}{|c|c|c|c|c|c|}
\hline $\begin{array}{c}\text { Input } \\
\text { Data }\end{array}$ & Multi & GMNMF & $\begin{array}{c}\text { CoNMF- } \\
\text { NMF }\end{array}$ & MVCC & OCMHAMVC \\
\hline
\end{tabular}




\begin{tabular}{|l|c|c|c|c|c|}
\hline 100 & 0.58 & 0.67 & 0.75 & 0.84 & 0.88 \\
\hline 200 & 0.64 & 0.71 & 0.78 & 0.87 & 0.91 \\
\hline 300 & 0.74 & 0.78 & 0.84 & 0.89 & 0.92 \\
\hline 400 & 0.78 & 0.84 & 0.87 & 0.89 & 0.94 \\
\hline 500 & 0.83 & 0.86 & 0.9 & 0.93 & 0.95 \\
\hline 600 & 0.85 & 0.89 & 0.92 & 0.94 & 0.97 \\
\hline
\end{tabular}

We can observe that table 2 describes recall values of different proposed approaches with constant consistently multi label clustering results. Figure 3 evaluates the performance of recall with different multi labeled html text content.

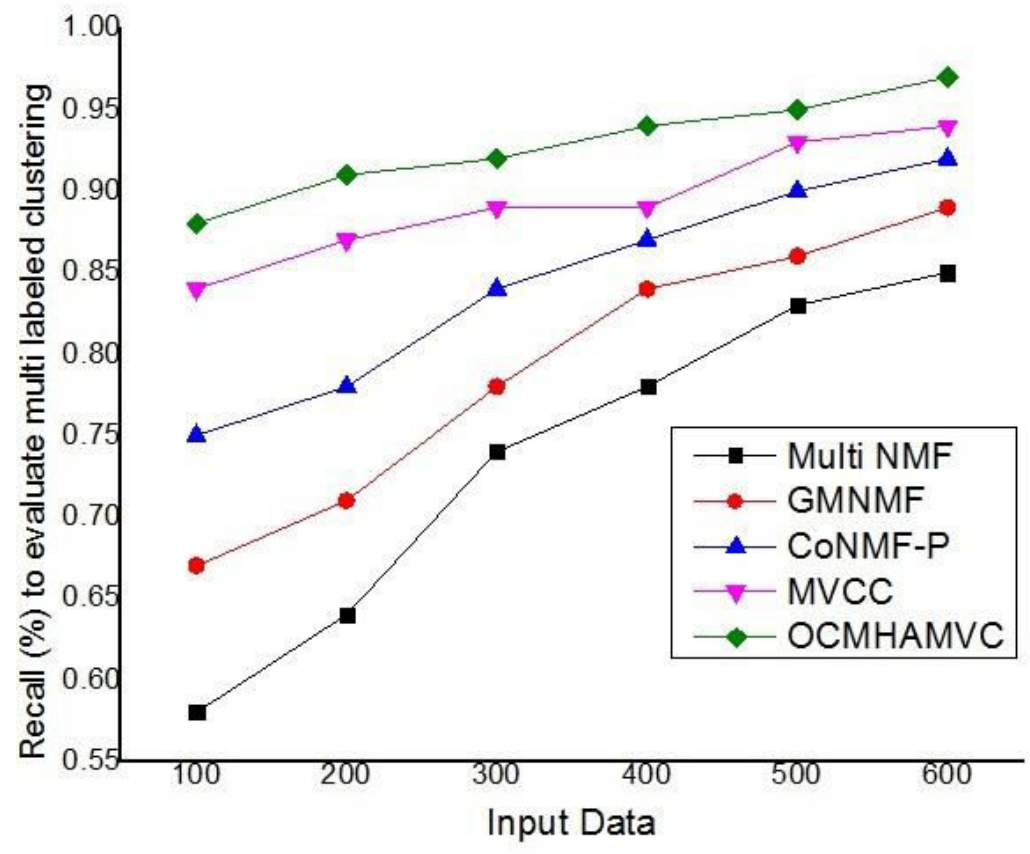

Figure 3 Performance of clustering results in recall.

As shown in figure 4, it describes the performance of f-score i.e. matched multi dimensional documents from html text documents, it shows that OCMHAMVC gives best results with comparison to traditional approaches when increase the documents then OCMHAMVC gives efficient cluster results with multi attributes. 


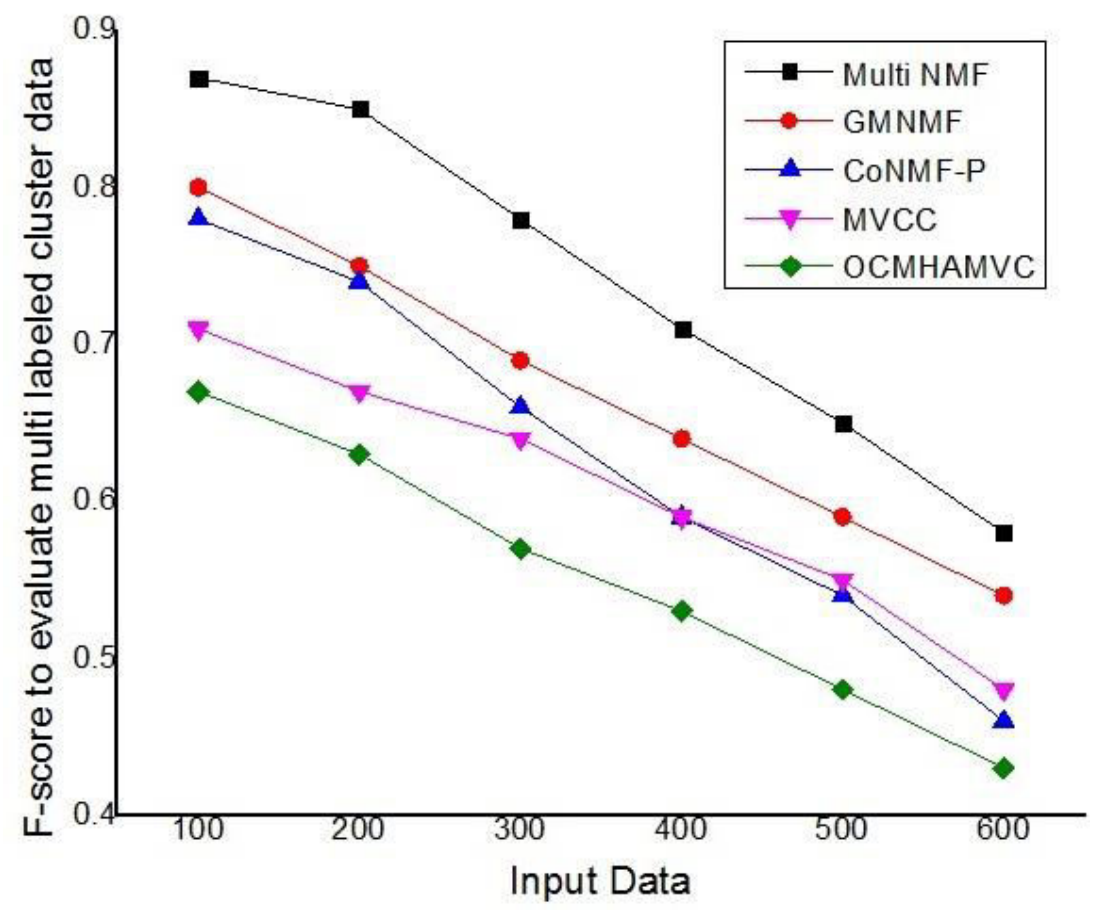

Figure 4 Performance of multi-dimensional clustering results in F-score.

Table 3 shows multi-dimensional clustering values with respect to F-score to increase the text documents relates to multi labels.

Table 3 Multi dimensional clustering results in F-score.

\begin{tabular}{|c|c|c|c|c|c|}
\hline $\begin{array}{c}\text { Input } \\
\text { Data }\end{array}$ & $\begin{array}{c}\text { Multi } \\
\text { NMF }\end{array}$ & GMNMF & $\begin{array}{c}\text { CoNMF- } \\
\mathbf{P}\end{array}$ & MVCC & OCMHAMVC \\
\hline 100 & 0.87 & 0.8 & 0.78 & 0.71 & 0.67 \\
\hline 200 & 0.85 & 0.75 & 0.74 & 0.67 & 0.63 \\
\hline 300 & 0.78 & 0.69 & 0.66 & 0.64 & 0.57 \\
\hline 400 & 0.71 & 0.64 & 0.59 & 0.59 & 0.53 \\
\hline 500 & 0.65 & 0.59 & 0.54 & 0.55 & 0.48 \\
\hline 600 & 0.58 & 0.54 & 0.46 & 0.48 & 0.43 \\
\hline
\end{tabular}

Figure 5 show the accuracy performance of five different clustering approaches applied on 100$500 \mathrm{html}$ text documents with high preference of multi labeled data collection. 


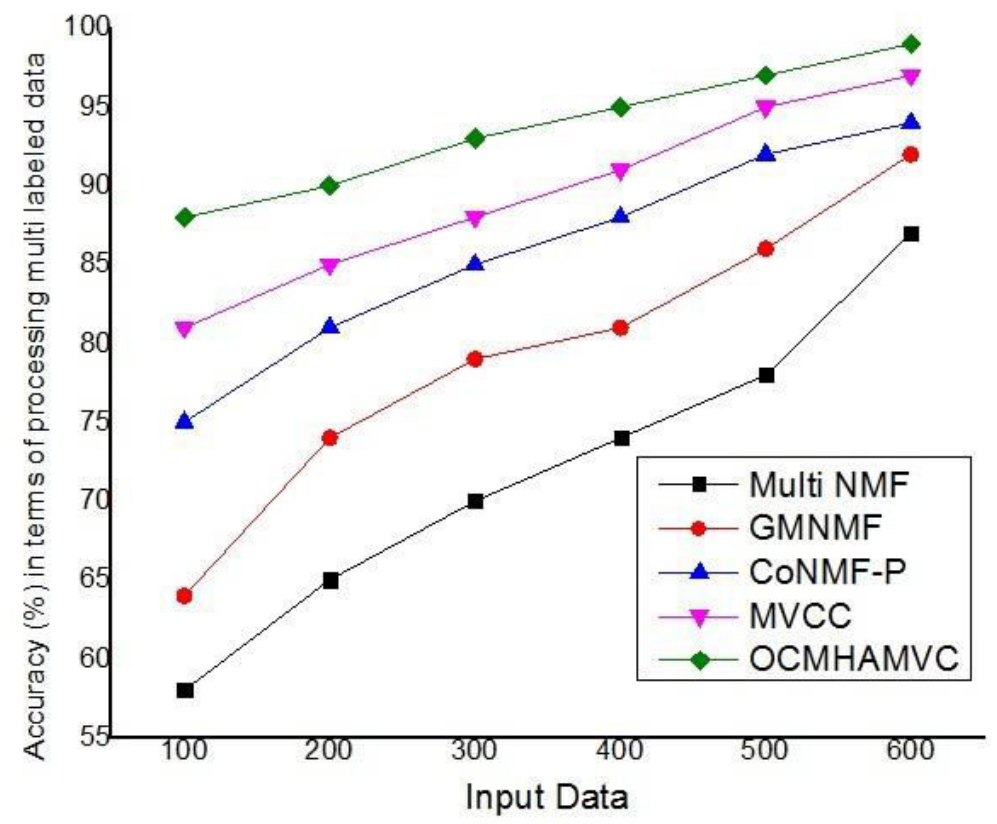

Figure 5 Performance of accuracy in multi -dimensional cluster results.

Clustering results are presented in different way based on accuracy values shown in table 4, for each data set present in row, best value is represented in bold and remaining values are second best results of different approaches.

Table 4 Multi-dimensional clustering results in accuracy.

\begin{tabular}{|c|c|c|c|c|c|}
\hline $\begin{array}{c}\text { Input } \\
\text { Data }\end{array}$ & $\begin{array}{c}\text { Multi } \\
\text { NMF }\end{array}$ & GMNMF & $\begin{array}{c}\text { CoNMF- } \\
\mathbf{P}\end{array}$ & MVCC & OCMHAMVC \\
\hline 100 & 58 & 64 & 75 & 81 & 88 \\
\hline 200 & 65 & 74 & 81 & 85 & 90 \\
\hline 300 & 70 & 79 & 85 & 88 & 93 \\
\hline 400 & 74 & 81 & 88 & 91 & 95 \\
\hline 500 & 78 & 86 & 92 & 95 & 97 \\
\hline 600 & 87 & 92 & 94 & 97 & 99 \\
\hline
\end{tabular}

As shown in figure 6, it describes the performance of time efficiency in retrieval matched multi dimensional documents from html text documents, it shows that OCMHAMVC gives best time results with comparison to traditional approaches when increase the documents then OCMHAMVC gives efficient cluster results i.e. less time to retrieve multi attributes relational documents which contain different domains. 


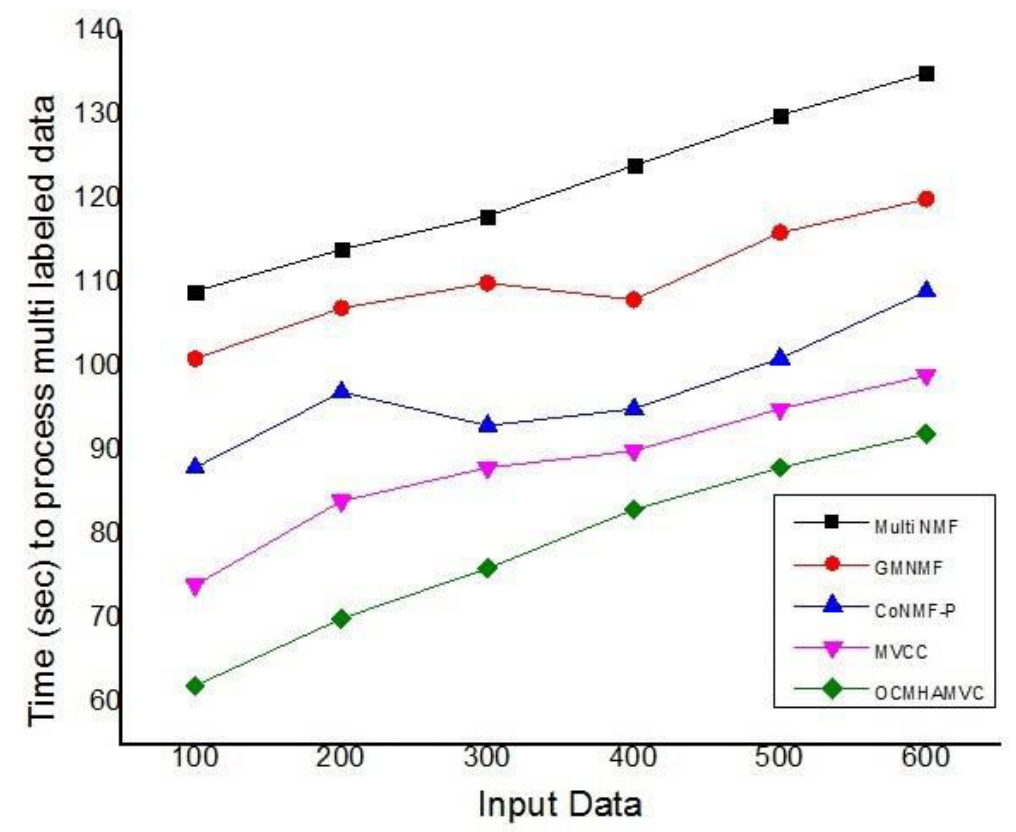

Figure 6 Performance of time in retrieval of multi-dimensional clustering results

Clustering results are presented in different way based on accuracy values shown in table 4, for each data set present in row, best value is represented in bold and remaining values are second best results of different approaches.

Table 5 Time efficiency values in formation of cluster with multi class labels

\begin{tabular}{|c|c|c|c|c|c|}
\hline $\begin{array}{c}\text { Input } \\
\text { Data }\end{array}$ & $\begin{array}{c}\text { Multi } \\
\text { NMF }\end{array}$ & GMNMF & $\begin{array}{c}\text { CoNMF- } \\
\mathbf{P}\end{array}$ & MVCC & OCMHAMVC \\
\hline 100 & 109 & 101 & 88 & 74 & 62 \\
\hline 200 & 114 & 107 & 97 & 84 & 70 \\
\hline 300 & 118 & 110 & 93 & 88 & 76 \\
\hline 400 & 124 & 108 & 95 & 90 & 83 \\
\hline 500 & 130 & 116 & 101 & 95 & 88 \\
\hline 600 & 135 & 120 & 109 & 99 & 92 \\
\hline
\end{tabular}

Figure 7 show performance of five different clustering approaches applied on 100-500 html text documents with high preference of multi labeled data collection in computational cost with preferable parameters. 


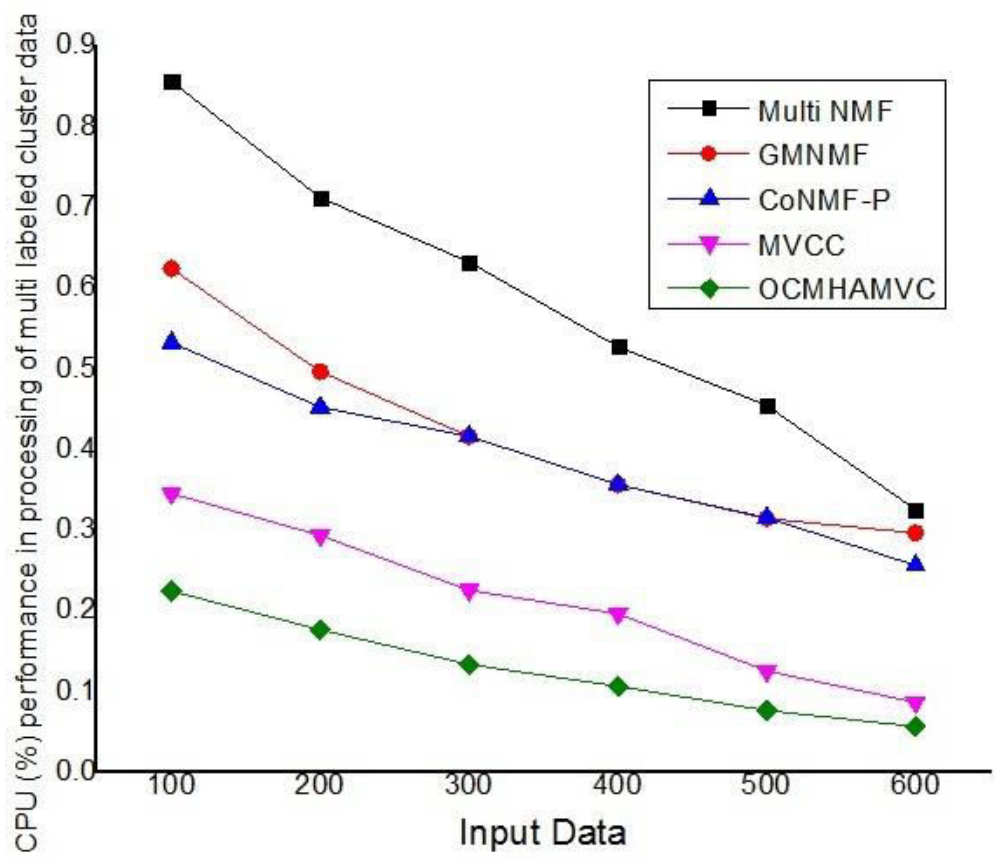

Figure 7Performance of computational cost in processing of CPU usage

Clustering results are presented in different way based on computational usage in CPU processing values shown in table 6 , for each data set present in row, best value is represented in bold and remaining values are second best results of different approaches.

Table 6 Computational cost values in multi-dimensional clustering results

\begin{tabular}{|c|c|c|c|c|c|}
\hline $\begin{array}{c}\text { Input } \\
\text { Data }\end{array}$ & $\begin{array}{c}\text { Multi } \\
\text { NMF }\end{array}$ & GMNMF & $\begin{array}{c}\text { CoNMF- } \\
\mathbf{P}\end{array}$ & MVCC & OCMHAMVC \\
\hline 100 & 0.856 & 0.624 & 0.532 & 0.345 & 0.224 \\
\hline 200 & 0.712 & 0.496 & 0.452 & 0.293 & 0.176 \\
\hline 300 & 0.632 & 0.416 & 0.416 & 0.225 & 0.133 \\
\hline 400 & 0.527 & 0.356 & 0.356 & 0.196 & 0.106 \\
\hline 500 & 0.454 & 0.314 & 0.315 & 0.125 & 0.076 \\
\hline 600 & 0.325 & 0.296 & 0.256 & 0.086 & 0.056 \\
\hline
\end{tabular}

As shown in figure 7 , it describes the performance memory utilization in multi dimensional documents from html text documents, it shows that OCMHAMVC gives best time results with comparison to traditional approaches when increase the documents then OCMHAMVC gives efficient cluster results i.e. less memory utilization to retrieve multi attributes relational documents which contain different domains 


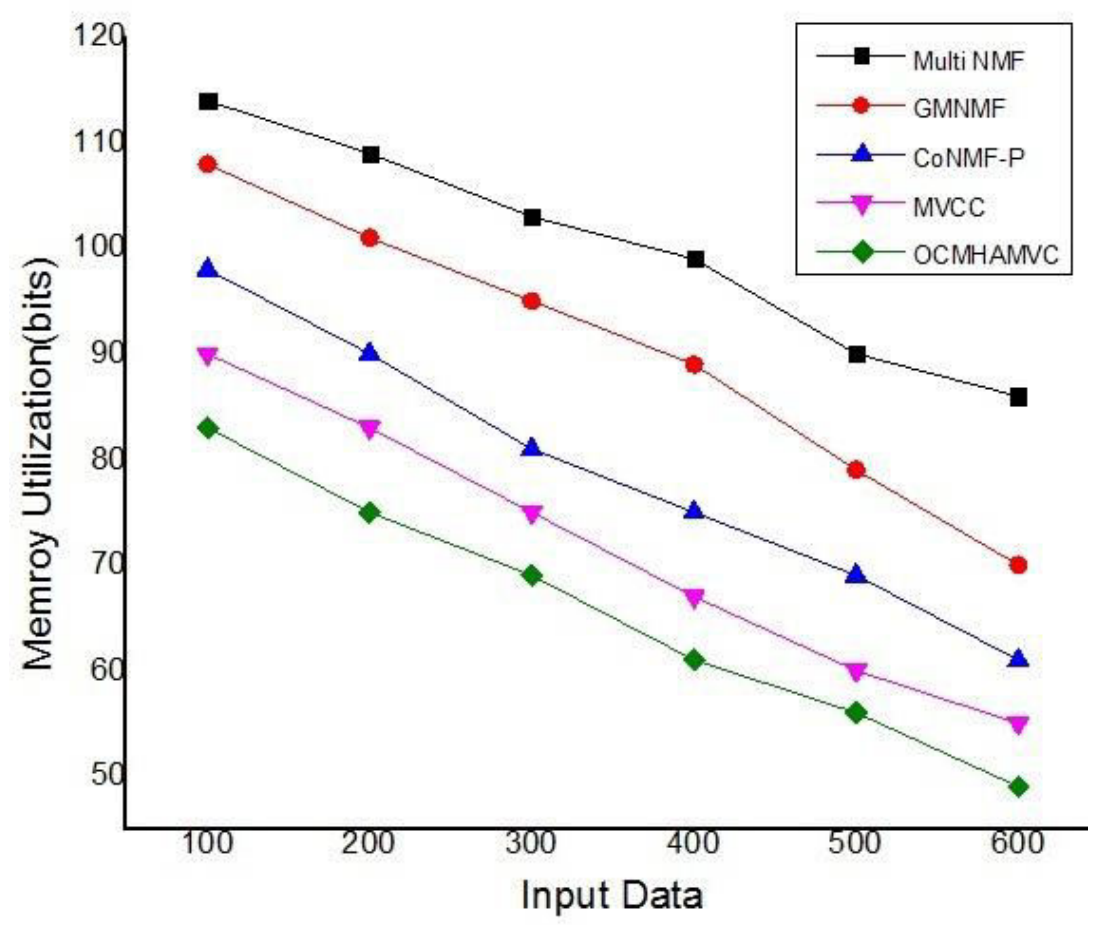

Figure 7 Performance evaluation of memory utilization in processing multi labeled data.

Table 3 shows multi-dimensional clustering values with respect to F-score to increase the text documents relates to multi labels

Table 7 Memory utilization values in multi-dimensional clustering

\begin{tabular}{|c|c|c|c|c|c|}
\hline $\begin{array}{c}\text { Input } \\
\text { Data }\end{array}$ & $\begin{array}{c}\text { Multi } \\
\text { NMF }\end{array}$ & GMNMF & $\begin{array}{c}\text { CoNMF- } \\
\mathbf{P}\end{array}$ & MVCC & OCMHAMVC \\
\hline 100 & 114 & 108 & 98 & 90 & 83 \\
\hline 200 & 109 & 101 & 90 & 83 & 75 \\
\hline 300 & 103 & 95 & 81 & 75 & 69 \\
\hline 400 & 99 & 89 & 75 & 67 & 61 \\
\hline 500 & 90 & 79 & 69 & 60 & 56 \\
\hline 600 & 86 & 70 & 61 & 55 & 49 \\
\hline
\end{tabular}

Based on experimental set up of proposed approach and other approaches results from above figures and tables. Through the basic convergence of OCMHAMVC, it applied on text oriented documents which contain different domains, it also satisfies the curve related text data. Objective functioning values in exploring documents with iterative functions, OCMHAMVC also find the efficient multi-dimensional clustering results with decrease of iterations applied on different text oriented documents. OCMHAMVC evaluates the computational performance with respect to time, precision, recall, accuracy, memory utilization and CPU computational cost in processing of multi dimensional documents. 


\section{Conclusion}

In this paper, we propose novel approach i.e. Orthogonal Constrained Meta Heuristic Adaptive Multi-View Clustering (OCMHAMVC) to represent data as a cluster with different categories with multi labeled dimensional data. With rapid development of labeled data, proposed approach first cluster the similar label data which are relevant to similar cluster prototypes. This cluster prototype consist unchanged similar labels with similar attribute relations associate with similar classes. For collecting similar cluster prototype proposed approach implement co-operative regularization with representative attributes to explore associative dimensions which are desirable with different views. Proposed approach evaluates low dimensional data using optimized matrix factorization (OMF) method and clusters the similar labeled sample data into prototype cluster of dimensional data. Proposed approach applied on different real time data sets and describes the effective performance evaluation with respect to state-of-the-art approaches. We also describe the performance of sampled class labeled cluster results with boundary relations compared to multi labeled dimensions. Further improvement of our proposed approach is would like to implement advanced machine learning multi dimensional clustering on different data stream environment.

\section{Conflict of interest:}

The authors declare that they have no conflict of interest.

\section{Data availability}

Data sharing not applicable to this article as no datasets were generated or analysed during the current study.

\section{Author Contribution:}

SK read and approved the final manuscript. MS read and approved the final manuscript. SK and MS have contributed equally.

\section{Funding}

No funding was received.

\section{Informed Consent Statement:}

Not Applicable

\section{References}

[1]Duc Thang Nguyen, Lihui Chen, "Clustering with Multiviewpoint-Based Similarity Measure". IEEE TRANSACTIONS ON KNOWLEDGE AND DATA ENGINEERING, VOL. 24, NO. 6, JUNE 2012 
[2] J. Liu, C. Wang, J. Gao, J. Han, Multi-view clustering via joint nonnegative matrix factorization, SDM (2013) 252-260.

[3] Z. Wang, X. Kong, H. Fu, M. Li, Y. Zhang, Feature extraction via multi-view non-negative matrix factorization with local graph regularization, in:International Conference on Image Processing, 2015, pp. 3500-3504.

[4] X. He, M.Y. Kan, P. Xie, X. Chen, Comment-based multi-view clustering of web 2.0 items, International World Wide Web Conferences (2014) 771-782.

[5] H. Wang, Y. Yang, T. Li, Multi-view clustering via concept factorization with local manifold regularization, in: International Conference on Data Mining,2016, pp. 1245-1250.

[6] Shaowei Wei,1 Jun Wang,1,* Guoxian Yu,1,2 Carlotta Domeniconi,3 Xiangliang Zhang2, "Multi-View Multiple Clusterings Using Deep Matrix Factorization", The Thirty-Fourth AAAI Conference on Artificial Intelligence (AAAI-20)

[7] Li, Z.; Tang, J.; and Mei, T. 2019. Deep collaborative embedding for social image understanding. TPAMI 41(9):2070-2083.

[8] Liu, X.; Zhu, X.; Li, M.; Wang, L.; Zhu, E.; Liu, T.; Kloft, M.; Shen, D.; Yin, J.; and Gao, W. 2019. Multiple kernel k-means with incomplete kernels. TPAMI 99(1):1-14.

[9] Luo, S.; Zhang, C.; Zhang,W.; and Cao, X. 2018. Consistent and specific multi-view subspace clustering. In AAAI, 3730- 3737.

[10] Mautz, D.; Ye, W.; Plant, C.; and B"ohm, C. 2018. Discovering non-redundant k-means clusterings in optimal subspaces. In KDD, 1973-1982.

[11] Nie, F.; Cai, G.; and Li, X. 2017. Multi-view clustering and semi-supervised classification with adaptive neighbours. In AAAI, 2408-2414.

[12] Tan, Q.; Yu, G.; Domeniconi, C.; Wang, J.; and Zhang, Z. 2018. Incomplete multi-view weak-label learning. In IJCAI, 2703-2709

[13] Wang, X.; Yu, G.; Domeniconi, C.; Wang, J.; Yu, Z.; and Zhang, Z. 2018. Multiple coclusterings. In ICDM, 1308- 1313

[14] Wang, X.; Wang, J.; Yu, G.; Domeniconi, C.; Xiao, G.; and Guo, M. 2019. Multiple independent subspace clusterings. In AAAI, 5353-5360.

[15] Yang, S., and Zhang, L. 2017. Non-redundant multiple clustering by nonnegative matrix factorization. Machine Learning 106(5):695-712

[16] Yao, S.; Yu, G.; Wang, X.; Wang, J.; Domeniconi, C.; and Guo, M. 2019a. Discovering multiple co-clusterings in subspaces,. In SDM, 423-431

[17] Yao, S.; Yu, G.; Wang, J.; Domeniconi, C.; and Zhang, X. 2019b. Multi-view multiple clustering. In IJCAI, 4121-4127.

[18] Zhao, H.; Ding, Z.; and Fu, Y. 2017. Multi-view clustering via deep matrix factorization. In AAAI, 2921-2927.

[19] Zong, L.; Zhang, X.; Zhao, L.; Yu, H.; and Zhao, Q. 2017. Multi-view clustering via multimanifold regularized nonnegative matrix factorization. Neural Networks 88:74-89.

[20] Shicheng Li ,1 Qinghua Liu ,1 Jiangyan Dai ,2 Wenle Wang ,1 Xiaolin Gui ,1 and Yugen Yi, "Adaptive-Weighted Multiview Deep Basis Matrix Factorization for Multimedia Data Analysis", Hindawi Wireless Communications and Mobile Computing Volume 2021, Article ID 5526479, 12 pages https://doi.org/10.1155/2021/5526479.

[21] J. H. Ahn, S. Kim, J. H. Oh, and S. Choi, "Multiple nonnegative-matrix factorization of dynamic PET images," in Proceedings of Asian Conference on Computer Vision, pp. 10091013, Jeju, Korea, 2004. 
[22] G. Trigeorgis, K. Bousmalis, S. Zafeiriou, and B. W. Schuller, "A deep matrix factorization method for learning attribute representations," IEEE Transactions on Pattern Analysis and Machine Intelligence, vol. 39, no. 3, pp. 417-429, 2016.

[23] Y. Zhao, H. Wang, and J. Pei, "Deep non-negative matrix factorization architecture based on underlying basis images learning," IEEE Transactions on Pattern Analysis and Machine Intelligence, vol. 43, no. 6, pp. 1897-1913, 2021.

[24] Y. Meng, R. Shang, F. Shang, L. Jiao, S. Yang, and R. Stolkin, "Semi-supervised graph regularized deep NMF with biorthogonal constraints for data representation," IEEE transactions on neural networks and learning systems, vol. 31, no. 9, pp. 3245-3258, 2020.

[25] M. Tong, Y. Chen, L. Ma, H. Bai, and X. Yue, "NMF with local constraint and deep NMF with temporal dependencies constraint for action recognition," Neural Computing and Applications, vol. 32, no. 9, pp. 4481-4505, 2020.

[26] J. Li, G. Zhou, Y. Qiu, Y. Wang, Y. Zhang, and S. Xie, "Deep graph regularized nonnegative matrix factorization for multiview clustering," Neurocomputing, vol. 390, pp. 108-116, 2020.

[27] Z. Shu, X. Wu, C. Hu, C. Z. You, and H. H. Fan, "Deep seminonnegative matrix factorization with elastic preserving for data representation," Multimedia Tools and Applications, vol. 80, no. 2, pp. 1707-1724, 2021.

[28] J. Zhao, X. Xie, X. Xu, and S. Sun, "Multi-view learning overview: recent progress and new challenges," Information Fusion, vol. 38, pp. 43-54, 2017.

[29] Y. Li, M. Yang, and Z. Zhang, "A survey of multi-view representation learning," IEEE Transactions on Knowledge and Data Engineering, vol. 31, no. 10, pp. 1863-1883, 2018.

[30] T. Hussain, K. Muhammad, W. Ding, J. Lloret, S. W. Baik, and V. H. C. de Albuquerque, "A comprehensive survey of multiview video summarization," Pattern Recognition, vol. 109, p. 107567, 2021.

[31] X. Xu, Y. Yang, C. Deng, and F. Nie, "Adaptive graph weighting for multi-view dimensionality reduction," Signal Processing, vol. 165, pp. 186-196, 2019.

[32] R. Zhang, F. Nie, X. Li, and X. Wei, "Feature selection with multi-view data: a survey," Information Fusion, vol. 50, pp. 158-167, 2019.

[33] P. Luo, J. Peng, Z. Guan, and J. Fan, "Dual regularized multiview non-negative matrix factorization for clustering," Neurocomputing, vol. 294, pp. 1-11, 2018.

[34] L. Bai, Y. Shao, Z. Wang, C. Li, Clustering by twin support vector machine and least square twin support vector classifier with uniform output coding, Knowl. Based Syst. 163 (2019) 227240.

[35] H.S. Seung, D.D. Lee, The manifold ways of perception, Science 290 (5500) (2000) 22682269.

[36] D.A. Spielman, Spectral graph theory and its applications, Found. Comput. Sci. (2007) 2938.

[37] D. Hidru, A. Goldenberg, Equinmf: graph regularized multiview nonnegative matrix factorization, Comput. Sci. (2014).

[38] L. Zong, X. Zhang, L. Zhao, H. Yu, Q. Zhao, Multi-view clustering via multi-manifold regularized non-negative matrix factorization, Neural Networks 88 (88) (2017) 74-89.

[39] Z. Guan, L. Zhang, J. Peng, J. Fan, Multi-view concept learning for data representation, IEEE Trans. Knowl. Data Eng. 27 (11) (2015) 3016-3028.

[40] C. Xu, Z. Guan, W. Zhao, Y. Niu, Q. Wang, Z. Wang, Deep multi-view concept learning, in: International Joint Conference on Artificial Intelligence, 2018, pp. 2898-2904. 
[41] J. Wang, X. Wang, F. Tian, C.H. Liu, H. Yu, Y. Liu, Adaptive multi-view semi-supervised nonnegative matrix factorization, International Conference on Neural Information Processing (2016) 435-444.

[42]Kolli, Srinivas, and M. Sreedevi. "Prototype analysis of different data mining classification and clustering approaches." ARPN Journal of Engineering and Applied Sciences 13.9 (2018): 3129-3135.

[43] Kolli, S., \&Sreedevi, M "A novel index based procedure to explore similar attribute similarity in uncertain categorical data." ARPN Journal of Engineering and Applied Sciences 14.12 (2019): 2266 -2272.

[44]Srinivas Kolli, M. Sreedevi. 2018. "Adaptive Clustering Approach to Handle Multi Similarity Index for Uncertain Categorical Data Streams". Jour of Adv Research in Dynamical \& Control Systems, Vol. 10, 04-Special Issue, 2018.

[45]Kolli, Srinivas. "A Novel Granularity Optimal Feature Selection based on Multi-Variant Clustering for High Dimensional Data." Turkish Journal of Computer and Mathematics Education (TURCOMAT) 12.3 (2021): 5051-5062.

[46]Srinivas Kolli, Peddarapu Rama Krishna \& Parvathala Balakesava Reddy“A NOVEL NLP AND MACHINE LEARNING BASED TEXT EXTRACTION APPROACH FROM ONLINE NEWS FEED”, ARPN Journal of Engineering and Applied Sciences 16.6 (2021): 679-685.

[47]Kolli, Srinivas, Parvathala Balakesavareddy, and M. Madhuri. "MACHINE LEARNING OPTIMAL DECISION PATH FINDER ALGORITHM FOR CUSTOMIZING TREATMENT IN THE HEALTHCARE INDUSTRY." Turkish Journal of Physiotherapy and Rehabilitation 32: 3(2021): 2453 - 2462

[48]Parvathala Balakesavareddy, Kolli, Srinivas, and Raswitha Bandi. " BRAIN STROKE DETECTION USING K-MEANS INTERFACED WITH FUZZY C-MEANS FOR IMPROVED ACCURACY AND SCANNING SPEED." Turkish Journal of Physiotherapy and Rehabilitation 32: 3(2021): 2463 - 2470 